\title{
Systemic Perspectives on National Infrastructure for a Sustainable, Resilient Net Zero Future
}

\author{
Tom Dolan*
}

Senior Research Associate, UKCRIC Coordination Node, University College London (CEGE UCL), London, United Kingdom

All aspects of Modern life are infrastructure-enabled. National infrastructure (NI) simultaneously: supports the realisation of societally beneficial outcomes; and determines the level of GHG emissions; air, water, noise pollution; production of solid waste and sewage. Therefore, all sustainability and resilience challenges are interdependent emergent properties arising directly or indirectly from National Infrastructure. $\mathrm{NI}$ is a systemically, societally, economically, globally significant leverage point. The systemic transformation of $\mathrm{NI}$ into a net zero enabling, resilience enhancing, sustainability supporting system is urgently needed to catalyse the speed, scale and breadth of synergistic action needed to achieve Net zero and tackle other sustainability and resilience challenges. Systemic perspectives on, and systemic characterisations of, $\mathrm{NI}$; its societal purpose; and the interdependent mechanisms that enable $\mathrm{NI}$ to fulfil its purpose are needed to support the required systemic transformation. This paper provides these. Ljubomir Jankovic,

Edited by University of Hertfordshire, United Kingdom

Reviewed by:

Timothy O. Adekunle, University of Hartford, United States

Silvio Carta, University of Hertfordshire, United Kingdom

${ }^{*}$ Correspondence:

Tom Dolan

thomas.dolan@ucl.ac.uk

Specialty section: This article was submitted to Urban Science,

a section of the journal Frontiers in Built Environment

Received: 03 August 2021 Accepted: 18 October 2021 Published: 15 November 2021

Citation:

Dolan T (2021) Systemic Perspectives on National Infrastructure for a Sustainable, Resilient Net Zero Future. Front. Built Environ. 7:752765. doi: 10.3389/fbuil.2021.752765
Keywords: conceptual framework, Emergen*, governance, outcomes, global warming, interdependent, synerg* transformation

\section{INTRODUCTION}

Global warming of at least $1.5^{\circ} \mathrm{C}$ is already inevitable (IPCC, 2018). Therefore, our lifestyles, societies, economies and the National Infrastructure that enables them must be systemically resilient to the disruptive impacts associated with global warming of at least $1.5^{\circ} \mathrm{C}$ (Dolan, 2018).

However, neither global warming of up to $4^{\circ} \mathrm{C}$, nor the need to be systemically resilient to the disruptive impacts of $4^{\circ} \mathrm{C}$ global warming, are yet inevitable. The future we choose remains in our hands (Figueres and Rivett-Carnac, 2020). Global warming can be limited to $1.5^{\circ} \mathrm{C}$ if Global Net Zero is achieved (i.e., if the sum of global GHG emissions is reduced to zero) by 2030 (IPCC, 2018).

Preventing further global warming (reducing global GHG emissions to net zero), and reducing the frequency, scale, intensity and duration of societal disruption caused by global warming (ensuring resilience to the impacts of global warming) are deeply interdependent Wicked system problems (Rittel and Webber, 1973; Dolan and Cosgrave, 2016). They must be addressed synergistically at a societal scale, using a diverse, long-term, collaborative, dynamic, multifaceted, multi-scale, cradle-tocradle and synergistic portfolio of systemically targeted interventions focused on transforming the wider system(s) from which they emerge.

The development of which will require the widespread adoption of system thinking principles to support: 1) the systemic diagnosis of Societal Leverage Points, System Archetypes/traps, systemic root causes, systemic constraints, and dissergistic, synergistic (and commensualistic) opportunities; 2 ) the identification and design of synergistic, systemically targeted interventions that exploit the above as opportunities to catalyse the necessary speed, scale and breadth of systemic transformation. 


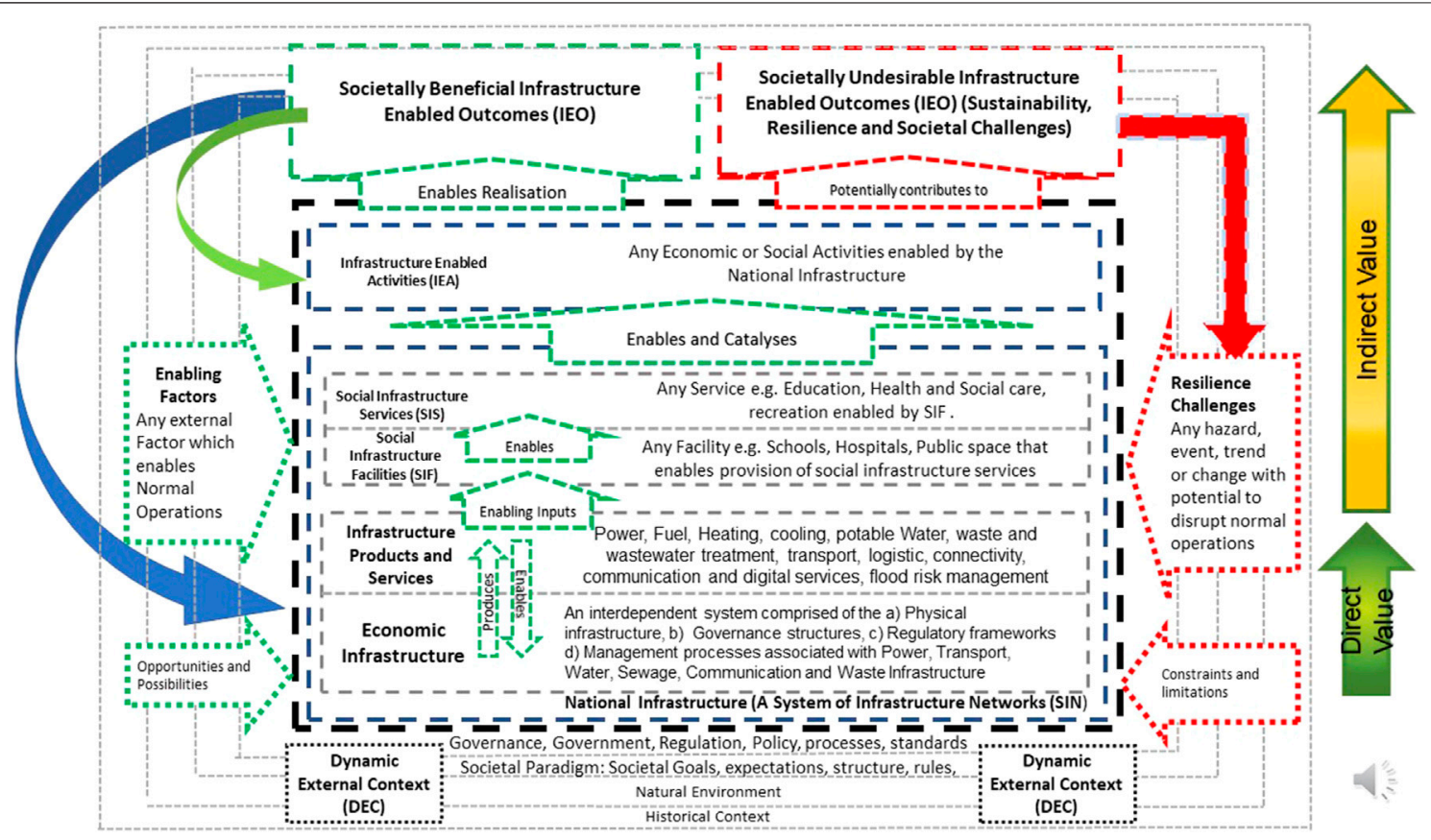

FIGURE 1 | Systemic characterisation of national infrastructure.

Across the globe, modern lifestyles, societies and national economies are increasingly enabled by, dependent upon, and could not function effectively in the absence of, National Infrastructure. In short, all aspects of modern life (including the above challenges) are infrastructure-enabled outcomes (IEO).

National Infrastructure (NI) is an open complex interdependent system comprised of the 1) Physical infrastructure networks, 2) Governance structures, 3) Regulatory frameworks 4) Management processes associated with the six economic infrastructure sectors of which it is comprised 5) Interdependencies within and between each of the above 6) Interdependencies with the Dynamic External Context (DEC) within which it is embedded (Figure 1).

The above National Infrastructure (NI) system (Figure 1) simultaneously:

- Produces a predictable flow of Infrastructure products and services (IP\&S), supports provision of social infrastructure services (SIS), catalyses wider societal and economic activity (IEA), enables realisation of the societally beneficial outcomes (IEO) expected by the society and economy it serves (Figure 1).

- Determines the level of GHG emissions, air, water, noise pollution, solid waste and sewage produced by the society and economy it serves.

- Determines the frequency, scale, intensity and duration of disruption to all levels of Figure 1, and hence the wider societal and economic impacts of, any given severity of resilience challenge.

Therefore, NI is a systemically, societally, economically and globally significant synergistic leverage point. With a significant role to play in enabling (or preventing) global progress toward a Sustainable, Resilient, Net Zero Future. NI can either:

- Enable (or impede) Net Zero: by catalysing (or impeding), progress toward Global Net Zero across all levels of the society, economy and value chains it enables (Figure 1).

- Enhance (or undermine) Sustainability: by catalysing (or impeding) society-wide reductions in the levels air, water, noise pollution, solid waste and sewage produced across all levels of the society and economy it enables (Figure 1).

- Enhance (Or undermine) Societal Resilience by mitigating (or multiplying) the frequency, scale, intensity, duration of wider societal and economic disruption caused by resilience challenges

However, at present National Infrastructure is as likely to impede as it is enable, to undermine as it is enhance, to multiply as it is mitigate. Therefore, systemic transformation of the NI that enables modern lifestyles, societies, economies into a net zero enabling, resilience enhancing, sustainability supporting system is urgently needed to catalyse synergistic society-wide progress.

The focus of this paper is on making the case that when viewed from a systemic perspective National Infrastructure is simultaneously a societal enabling system, a driver of Sustainability Challenges, and a systemically, societally, economically, globally significant leverage point which if systemically transformed can unlock and catalyse societal wide progress toward a Sustainable Resilient Net Zero future. Three main novel outputs are presented to support this case. 
TABLE 1 | Systemic perspectives on national infrastructure as a societal leverage point for a sustainable resilient net zero future.

SP1 Systemic characterisation: National infrastructure (NI) is an open complex interdependent system comprised of the 1) Physical infrastructure networks, 2) Governance structures, 3) Regulatory frameworks 4) Management processes associated with the six economic infrastructure sectors of which it is comprised 5) Interdependencies within and between each of the above 6) Interdependencies with the Dynamic External Context (DEC) within which it is embedded

SP2 Modern lifestyles, societies and national economies are emergent infrastructure-enabled outcomes (IEO) uniquely enabled by National Infrastructure

SP3 Purpose: The societal purpose of National Infrastructure is to enable realisation of the societal and economic benefits (Infrastructure Enabled Outcomes IEO) expected by the society and economy it serves (Figure 1)

SP4 Normal Operations and Purpose: NI fulfils its purpose indirectly, as part of a wider system of systems (Figure 1). The normal operations of NI produce: a predictable flow of infrastructure products and services (IP\&S), which in turn support, catalyse and enable: the operation of social infrastructure facilities, the provision of social infrastructure services (SIS); wider societal and economic activity (IEA) and the realisation of societally beneficial IEO (all other levels of Figure 1). All of which are interdependent emergent properties arise directly or indirectly from National Infrastructure (Figure 1)

SP5 Enabled by the DEC: The Normal operation of NI, and all levels of Figure 1, are enabled by and dependent upon a predictable flow of enabling factors (EF) from the DEC within. Which they are embedded

SP6 A Finely Balanced Cascade Success: The Normal operations of National Infrastructure and the whole system represented in Figure $\mathbf{1}$ are a finally balanced cascade success. Intrinsically vulnerable to interdependence related disruptions (cascade, common cause or escalating failure) initiated by any event (resilience challenges) or systemic trends, that disrupts the enabling flows on which it depends

SP7 GHG Emissions and Sustainability Challenges: Not all IEO are intentionally enabled or societally beneficial outcomes. Sustainability challenges, such as the level of GHG Emissions, air pollution, water pollution, noise pollution, solid waste and sewage produced by any infrastructure-enabled nation are also IEOs, arising directly or indirectly from National Infrastructure (Figure 1)

SP8 Interdependent Emergent Properties: Societally beneficial IEO, GHG emissions, other sustainability challenges, resilience challenges and other undesirable IEO are deeply interdependent, tightly coupled, emergent properties all of which arise directly or indirectly from National Infrastructure (Figure 1)

SP9 Deeply Interdependent Wicked Challenges: Therefore, undesirable IEOS (e.g., GHG Emissions, Sustainability Challenges, Resilience Challenges) are deeply interdependent challenges best resolved synergistically through a diverse, long-term, collaborative, dynamic, multifaceted, multi-scale, cradle-to-cradle and synergistic portfolio of systemically targeted interventions focused on transforming the wider system(s) (Figure 1) from which they emerge

SP10 Societal and Economic Value: NI is both a value generating and a value enabling system. The societal and economic value of National Infrastructure to the citizens, households, communities, towns, cities, societies and economies it serves encompasses both: the total value of the IP\&S it produces (value generated); and the value of the SIS, IEA and IEO it enables (Figure 1)

SP11 The Systemic Resilience of National Infrastructure: Is a dynamic, emergent and intrinsic characteristic of the NI system characterised in SP 1. It encompasses the degree to which $\mathrm{NI}$ is able to reduce the frequency, scale, speed, and duration of disruption to normal operations caused by any level of resilience challenges

SP12 Resilience Challenges: Originate in the DEC (Figure 1) and include "all sustainability challenges, actions, events, decisions or trends which have the potential to impede the production, or availability, of a predictable flow of one or more IP\&S; and thus disrupt the Normal operations of National Infrastructure." (Dolan, 2021)

SP13 Societal and Economic Impacts: The systemic resilience of National Infrastructure is a critical determinant of the frequency, scale, intensity and duration of disruption to the SIF, SIS, IEA and IEO it enables (all other levels of Figure 1), and hence the wider societal and economic impacts of, any given severity of resilience challenge

Highly resilient National Infrastructure can reduce the societal and economic impact of resilience challenges; whereas, low resilience National Infrastructure will have the opposite impact.(Dolan, 2021)

SP14 Societal and Economic Value of Highly Resilient NI: The value of highly resilient NI is directly related to the societal and economic value of avoiding disruption to the societal value described in SP10 and avoiding the societal and impacts in SP13

SP15 Systems Thinking Principles: Drawing on (Meadows, 2009), a sustainable, resilient, Net Zero Future will require the widespread adoption of System Thinking principles and an historic perspective to support: 1) the systemic diagnosis of Societal Leverage Points, System Archetypes/traps, systemic root causes, systemic constraints, and dissergistic, synergistic (and commensualistic) opportunities; 2) the identification and design of synergistic, systemically targeted interventions that exploit the above as opportunities to catalyse the necessary speed, scale and breadth of systemic transformation

SP16 The Role of National Infrastructure: NI has a significant role to play in enabling (or preventing) a Sustainable, Resilient, Net Zero Future. It is a systemically, societally, economically and globally significant synergistic leverage point. NI can either:

- Enhance (Or undermine) Societal Resilience: By mitigating (or multiplying) the frequency, scale, intensity, duration of wider societal and economic disruption caused by resilience challenges

- Enable (or impede) Net Zero: By catalysing (or impeding), progress toward Global Net Zero across all levels of the society, economy and value chains it enables (Figure 1)

- Enhance (or undermine) Sustainability: By catalysing (or impeding) society-wide reductions in the levels air, water, noise pollution, solid waste and sewage produced across all levels of the society and economy it enables (Figure 1)

SP17 The case for Systemic Transformation: The Systemic Transformation of National Infrastructure into a net zero enabling, sustainability and resilience enhancing system is urgently needed to catalyse synergistic society-wide progress toward a sustainable, net zero, resilient economy (Dolan, 2019)

Whereas, the continued absence of these qualities from NI will exacerbate the scale and impact of global warming, and impede societal progress toward a sustainable net zero resilient future

SP18 A Globally Significant Leverage Point: The ST of NI in systemic perspective 17 is a unilateral action that can be applied by all nations globally. A globally significant leverage point to support mitigation of the causes of, and enhance societal resilience to, all sustainability challenges

(Continued on following page) 
TABLE 1 | (Continued) Systemic perspectives on national infrastructure as a societal leverage point for a sustainable resilient net zero future.

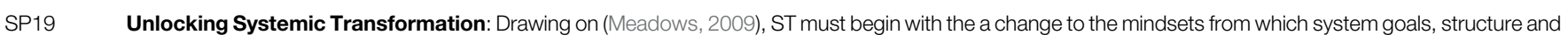
rules emerge (Paradigms); a compelling vision of the societal and economic benefits of NI with the qualities "net zero enabling," "sustainability supporting," "resilience enhancing"; and explicit integration of the above qualities into: 1) the stated purpose of National Infrastructure; and 2) All Governance structures that influence National Infrastructure decision making processes

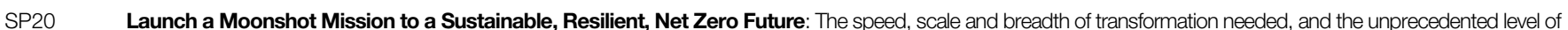
interdisciplinary, cross-sectoral, society-wide collaboration will requires the "Launch of a Moonshot (CST, 2020) Mission (Mazzucato, 2017; 2018a; 2018b; 2018c, 2021) tasked with establishing a diverse long-term, collaborative, dynamic, multifaceted, multi-scale, cradle-to-cradle and synergistic portfolio of systemically targeted interventions"

The first is a novel systemic characterisation of National Infrastructure (See Systemic Perspective 1-Table 1). The second is a novel conceptual model (Figure 1), supporting terminology and narrative to illustrate, justify and elaborate upon the above systemic characterisation of NI. The third is a set of 20 systemic perspectives that both support and draw upon the above characterisation of National Infrastructure.

The Discussion section of the paper elaborates upon and justifies the significance and complementarity of each of the above outputs. Specifically, the Discussion section explores the interdependence between sustainability challenges and resilience challenges and the critical importance of NI that is systemically resilient to resilience challenges; it explores the societal value proposition of net zero, resilience enhancing, sustainability supporting NI; it draws on the work of Meadows (2009, Mazzucato (2017, 2018a, 2018b, 2018c, 2021) and the CST (2020) to explore how the diverse long-term, collaborative, dynamic, multifaceted, multi-scale, cradle-to-cradle and synergistic portfolio of systemically targeted interventions required to transform NI into a net zero enabling, sustainability support, resilience enhancing system could most effectively be achieved.

\section{METHODS}

The research published in this paper is a novel synthesis of the key concepts, emergent themes and conclusion from the authors previously published research on the topic of National Infrastructure, undertaken as part of two EPSRC (Grants EP/K012347/1 and EP/R017727/1).

The Conceptual model and characterisation of National Infrastructure (Figure 1), the systemic perspectives outlined in Table 1 and the topics covered in the Discussion are all novel outputs from this process of interpretive synthesis undertaken as part of this research.

Each draws upon multiple sources, collaborations with infrastructure practitioners, published work undertaken with or on behalf collaborators and responses to a number of UK consultations on systemic topics connected to the role of National Infrastructure in enabling all aspects of modern society.

Furthermore, each has been has been shared, tested iterated upon and refined through a continuous process of interpretive synthesis prior to publication.

\section{MAIN RESULTS}

The main outputs from this research are a novel systemic characterisation of National Infrastructure as a complex interdependent system, a conceptual model of National Infrastructure (Figure 1); systemic characterisation of National Infrastructure on which Figure $\mathbf{1}$ is based, the systemic perspectives on the role of National infrastructure as a societally significant leverage point for a sustainable resilient net zero future (Table 1) and the systemic narrative that supports justifies and elaborates upon the significance of the Figure 1 and Table 1 (see discussion section).

\section{DISCUSSION}

\section{Systemic Characterisation of National Infrastructure}

National Infrastructure (NI) is an open complex interdependent system comprised of the 1) Physical infrastructure networks, 2) Governance structures, 3) Regulatory frameworks 4) Management processes associated with the six economic infrastructure sectors of which it is comprised 5) Interdependencies within and between each of the above 6) Interdependencies with the Dynamic External Context (DEC) within which it is embedded. (Systemic Perspective 1 - Table 1).

The conceptual model in Figure $\mathbf{1}$ and the systemic narrative that supports it draw upon an extensive literature review of potential systemic approaches to the characterisation of National Infrastructure (Dolan, 2017), a literature review of the application Rinaldi's six interdependency dimensions (Rinaldi et al., 2001) to National Infrastructure (Dolan and Street, 2019) and an extensive body of work on resilience.

Dolan, (2017) observed that National Infrastructure can be characterised systemically as a complex adaptive system (Oughton et al., 2018), an interdependent system (Rinaldi et al., 2001); a sociotechnical system (STS); a high-risk (tightly coupled and interactively complex) system (Perrow, 2011); Large Technical Systems (LTS) (Hughes, 1989), an intentional and dynamic built system (Hollnagel, 2014), a complex engineered system (Punzo et al., 2020) or as a network of conversion points (Varga et al., 2014). Based on which, Dolan, (2017) concluded that each approach offers useful insights relevant to developing systemic understanding of the characteristics, performance, resilience and societal value of National Infrastructure.

The six economic infrastructure sectors of which National Infrastructure is comprised (Figure 1) are energy, transport, water and wastewater (drainage and sewerage), waste, flood risk management and digital communications (HM Treasury, 2017). 
Although typically governed, regulated and managed separately, these sectors are in practise deeply interdependent (Beckford, 2009; Carhart and Rosenberg, 2016; Rosenberg et al., 2014; Royal Academy of Engineering (Great Britain) and Engineering the Future (Organization), 2011). Each shares at least one continuous and absolute interdependence with at least one other (Beckford, 2009). Therefore, in practise, none can operate without a predictable flow of Infrastructure Products and Services (IP\&S) from at least one other. Moreover, no individual IP\&S is produced by a single sector operating in isolation. Therefore, disruption to one sector is highly likely to result in disruption to all sectors.

The purpose of National Infrastructure is to enable realisation of the societally beneficial outcomes expected by the society it serves. (Systemic Perspective 3 - Table 1).

NI fulfils this purpose indirectly, and as part of a wider system of systems (Figure 1) by producing a predictable flow of infrastructure products and services (IP\&S), which in turn support, catalyse and enable all other levels of Figure $\mathbf{1}$. (Systemic Perspective 4 - Table 1).

These IP\&S include Power, Fuel, heating, cooling, potable water, waste and sewage treatment, transport, logistics, connectivity, communication and digital services, flood risk management.

The predictable flow of IP\&S produced by National Infrastructure is an emergent property arising from the normal operations of the whole interdependent system. Moreover, the normal operations of the whole system is itself also an emergent property, arising from:

1. A predictable flow of enabling factors (EF) from the dynamic external context (DEC) within which it is embedded

2. A predictable flow of IP\&S (NB: National Infrastructure is an autocatalytic system because it both depends upon and produces a predictable flow of IP\&S)

3. Systemic resilience to the disruptive impacts caused by disruption to either of the above flows.

The predictable flow of IP\&S produced by National Infrastructure provides a flow of enabling inputs to make possible:

1. The operation of social infrastructure facilities (SIF) and provision of social infrastructure services (SIS) (Figure 1) and

2. Infrastructure enabled activities (IEA). Where IEA include all other social or economic activities uniquely enabled by National Infrastructure.

Therefore, both SIS and IEA are emergent properties uniquely enabled by factors 1) and 3) (Systemic Perspective 4 - Table 1).

Together National Infrastructure, SIS and IEA make possible infrastructure enabled outcomes (IEO). IEO are outcomes that are dependent upon, uniquely enabled by and could not happen in the absence of:

$1-3$ above and

4. The provision of one or more social infrastructure services (SIS), and/or;
5. One or more infrastructure enabled activities (IEA).

Therefore, IEO are emergent properties made possible by 1)-5). (Systemic Perspective 4 - Table 1).

\section{The Dynamic External Context}

All levels of Figure 1 are deeply embedded within, and made possible by a dynamic external context (DEC) (Systemic Perspective 5 - Table 1).

The DEC is itself, an open interdependent system comprised of, and shaped by, governance, government, regulation, policy, processes, standards, societal goals, expectations, structures, rules, the natural environment and the historical context.

In the context of National Infrastructure, the DEC is relevant as both a provider of a predictable flow of enabling factors (EF) and a source of resilience challenges (discussed later).

The term enabling factor (EF) refers to any aspect(s) of the DEC that enables the normal operations of National Infrastructure. Examples of EF include, but are not limited to: people, supply chains, logistics, incumbent technologies, services, inputs, resources, technology, stable ambient conditions, natural processes, natural capital, ecosystem services, markets, legal system, standards and codes of practise.

A predictable flow of enabling factors (EF) from the DEC makes possible the normal operations of National Infrastructure, the production of a predictable flow of IP\&S and the role played by National Infrastructure in enabling the higher levels of Figure 1 (SIF, SIS, IEA, and IEO) (Systemic Perspective 5 Table 1).

Therefore, the impact of disruption to the flow of (EF) has the potential to disrupt all other levels of Figure 1. In the context of understanding the impacts of global warming on the performance of NI and society, and the resilience of National Infrastructure to global warming driven resilience challenges (GWDRC), the most significant EF from the DEC are stable ambient conditions, natural processes, natural capital and ecosystem services (Edwards, 2003; Helm, 2020).

\section{Cascade Success-Cascade Failure}

The normal operation of NI, and all levels of the system represented in Figure $\mathbf{1}$ is a finally balanced cascade success (Dolan, 2021), enabled by aspects 1)-6) of the open complex interdependent National Infrastructure (NI) system characterised in Systemic Perspective 1 (Table 1) and aspects 1)-5) outlined above.

However, the whole system is inherently vulnerable to interdependence related disruptions (cascade, common cause or escalating failure) (Rinaldi et al., 2001; Dolan and Street, 2019). Because any change intentionally or otherwise to system structures 1)-6); or any disruption effecting 1)-5) has the potential to modify system performance and/or disrupt the production or availability of one or more IP\&S. Thus potentially disrupting the normal operations of NI (Systemic Perspective 6 - Table 1).

Causing, further disruption to the production or availability of IP\&S, further disrupting normal operations of NI. All of which ultimately impair the ability of NI to enable the higher levels of Figure 1. 
The significance of this intrinsic vulnerability is explored further in a later section of this discussion.

\section{GHG Emissions and Sustainability Challenges}

All aspects of modern societies are directly or indirectly enabled by National Infrastructure. (Systemic Perspective 2 - Table $\mathbf{1}$ ).

However, not all National Infrastructure enabled outcomes (IEO) are societally beneficial or intentional outcomes. The normal operation of National infrastructure also drives the emergence of societally undesirable IEO (Figure 1).

Sustainability Challenges such as the level of GHG emissions, air, water, noise pollution, solid waste and sewage produced by an infrastructure-enabled nation are interdependent emergent properties (unintentional outcomes) arising directly or indirectly from NI (Figure 1). They can emerge directly from the IP\&S it produces and/or indirectly from the SIS provision it supports; wider IEA it catalyses; and the societally beneficial outcomes (IEO), lifestyles, societies and economies it enables. For example, the GHG emissions from National Infrastructure are a significant determinant of the level of GHG emissions arising from the wider SIF, SIS, IEA, and IEO (Figure 1) NI enables (Systemic Perspective 7 - Table 1).

Using terminology from the GHG Protocol (World Business Council for Sustainable Development, and World Resources Institute (Eds.), 2004), the scope 1 GHG emissions of National Infrastructure determine the Scope 2 and Scope 3 emissions of the SIF, SIS, IEA, and IEO they enable.

Therefore, National Infrastructure is a societally significant systemic leverage point capable of catalysing GHG emission reductions across all levels of the value chains it enables (Figure 1). Thus, driving progress toward a Net Zero Economy (Systemic Perspective 16ii - Table 1).

Sustainability challenges are emergent properties. They are caused by no one party in isolation. Rather, they emerge as a consequence of interdependencies between multiple system components-including the DEC (Figure 1) within which they are embedded (Systemic Perspective 8 - Table 1).

Whilst Societally beneficial IEO (e.g., wellbeing, quality of life and economic prosperity) and societally undesirable IEO (e.g., GHG Emissions, other sustainability challenges) are both emergent properties arising directly or indirectly from National Infrastructure (Figure 1). A key difference between the two is that societally beneficial IEO are enabled intentionally. Whereas, Sustainability challenges (and other undesirable IEO) emerge unintentionally, are often regarded as acceptable trade-offs or externalities, and preventing them is not an explicit priority of National infrastructure governance.

Nevertheless, Societally beneficial IEO, GHG emissions, other sustainability challenges, resilience challenges and other undesirable IEO are deeply interdependent, tightly coupled, emergent properties all of which arise directly or indirectly from National Infrastructure (Figure 1) (Systemic Perspective 8 - Table 1).

Therefore, GHG emissions, other sustainability challenges, resilience challenges and other undesirable IEO are deeply interdependent wicked challenges best resolved synergistically through a diverse, long-term, collaborative, dynamic, multifaceted, multi-scale, cradle-to-cradle and synergistic portfolio of systemically targeted interventions focused on transforming the wider system(s) (Figure 1) from which they emerge. (Systemic Perspective 9 - Table 1).

\section{Resilience and Resilience Challenges}

\begin{abstract}
"The Systemic Resilience of National Infrastructure: is a dynamic, emergent and intrinsic characteristic of the NI system characterised in SP 1. It encompasses the degree to which NI is able to reduce the frequency, scale, speed, and duration of disruptive impacts initiated by resilience challenges." (adapted from Dolan, 2020; Dolan, 2021). (Systemic Perspective 11 - Table 1).

Where, "resilience challenges originate in the DEC (Figure 1) and include all sustainability challenges, actions, events, decisions or trends which have the potential to disrupt the ability of National Infrastructure to produce a predictable flow of one or more IP¿S; and thus disrupt the Normal operations of National Infrastructure." (adapted from Dolan, 2021). (Systemic Perspective 12 - Table 1)
\end{abstract}

Resilience challenges are significant because, the whole system represented in Figure $\mathbf{1}$ is a finally balanced cascade success enabled by 1)-5). It is, therefore, intrinsically vulnerable to disruption to the production or availability of one or more IP\&S. (Systemic Perspective 6 - Table 1).

It is therefore, important to understand the systemic mechanisms through which resilience challenges could potentially disrupt the production or availability of IP\&S. These could occur via one, or any combination of, the following mechanisms.

- Sudden disruption to the supply of one or more EF

- Long term trends in the supply of one or more EF

- Direct physical damage to one or more NI system component

- Direct physical disruption to one or more NI system component

- Rapid changes in short term demand for IP\&S

- Increased frequency of NI operation outside of design thresholds (Dolan, 2021)

\section{Translating Sustainability Challenges Into Resilience Challenges}

Sustainability challenges cause the emergence of resilience challenges. National Infrastructure must be resilient to the disruptive impacts of these, and other, resilience challenges if it is produce the predictable flow of IP\&S required, to enable the higher levels of Figure 1 (i.e., support SIS provision, catalyse wider IEA) and fulfil its purpose (i.e., enable realisation of societally beneficial Outcomes).

For example, GHG emissions (a sustainability challenge) gives rise to global warming driven resilience challenges (GWDRC). In-depth systemic understanding is needed to assess what these 
GWDRC are likely to be; and how the intensity of GWDRC will change with higher levels of Global warming. The GWDRC of greatest significance to National Infrastructure are likely to include.

- Long-term changes to ambient operating conditions

- Increased frequency, intensity, duration, clustering of extreme events

- Disruption (temporary or permanent) to the flow of ecosystem services enabled by Natural Capital

- Temporary or permanent disruption to the available operating capacity of individual system components.

- Disruption to the supply of products or services

- Changes to the type, quality, quantity, timing, location of demand for IP\&S

- The creation of pre-existing stresses and other sources of latent vulnerabilities

- Unforeseen increases in the relative criticality of individual system components.

Therefore, whilst the magnitude and severity of GWDRC is determined by whether global Net Zero GHG emissions is achieved (and by when). The subsequent frequency, scale, intensity and duration of disruption to National Infrastructure and wider society and as a result of any given severity of GWDRC is determined by the degree to which National Infrastructure is intrinsically resilient (or not).

Moreover, the frequency, scale, intensity and duration of disruption to the other levels of Figure 1 (wider society and the economy) caused by GWDRC is also a function of National Infrastructure resilience. Therefore, the societal and economic impacts of GWDRC can be reduced by highly resilient National Infrastructure (Systemic Perspective 13 - Table 1).

More broadly, this can be generalised for all Resilience challenges. Therefore, the systemic resilience of National Infrastructure is a critical determinant of the frequency, scale, intensity and duration of disruption to the SIF, SIS, IEA, and IEO it enables (all other levels of Figure 1), and hence the wider societal and economic impacts of, any given severity of resilience challenge.

Highly resilient National Infrastructure, is disrupted less frequently can reduce the societal and economic impact of resilience challenges; Whereas, low resilience National Infrastructure will have the opposite impact (Dolan, 2021). (Systemic Perspective 13 - Table 1)

Therefore, NI is a societally significant leverage point capable of either mitigating or multiplying the frequency, scale, intensity, duration of wider societal and economic disruption caused by resilience challenges. (Systemic Perspective 16i - Table 1).

Figure 1 provides a systemic perspective to support enhanced understanding of the mechanisms, system structures and interdependencies:

1. That enable the normal operations of National Infrastructure.

2. Through which the normal operations of National Infrastructure enables social infrastructure service provision, enables wider societal and economic activity (IEA) and ultimately supports realisation of societally beneficial outcomes (IEO)

3. Through which sustainability challenges translate into resilience challenges

4. Through which resilience challenges disrupt National Infrastructure performance

5. Through which disruption to National Infrastructure translates into disruption to wider societal and economic activity (IEA.)

A systemic perspective on 1)-5) can support analysis of the societal and economic value of enhancing the systemic resilience of National Infrastructure (Systemic Perspective 14 - Table 1). These benefits include the reduction in the frequency, scale, intensity and duration of 3,4 , and 5 ; which thus maximise the societal value of National Infrastructure to other levels of (Figure 1). Thus illustrating the potential societal and economic value of collaborative action to increase the resilience of National Infrastructure and strengthening the case that National Infrastructure is a powerful societal leverage point for targeted interventions that enhance society-wide resilience to Resilience Challenges. (Systemic Perspective 16i - Table 1).

Moreover, a systemic perspective on 1)-6) can support analysis of the societal value of avoiding any actions, events, decisions or trends within National Infrastructure that reduce the intrinsic resilience of National Infrastructure to resilience challenges by causing one or any combination of the following:

- Increased likelihood of a initial single point of failure (SPOF) occurring

- Increased likelihood of an initial SPOF becoming a Cascade Failure

- An increase in the number, frequency and criticality of interdependencies

- Increased criticality of any individual components

- System operation outside of design specifications more frequent and/or longer duration

- Removes spare capacity/headroom/redundancy

- Optimise the system to inappropriate criteria

\section{A Societal Value Perspective}

$\mathrm{NI}$ is both a value generating and a value enabling system. The societal and economic value of National Infrastructure to the citizens, households, communities, towns, cities, societies and economies it serves (wider societal value) encompasses both: the direct value of the IP\&S it produces (value generated); and the indirect value of the SIS, IEA and IEO it enables (value enabled) (Figure 1). (Systemic Perspective 10 - Table 1)

National Infrastructure fulfils its purpose (i.e., enables societally beneficial outcomes) indirectly via the predictable flow of IP\&S it produces (Figure 1). (Systemic Perspective 4 Table 1)

Direct Value includes only the revenue directly generated from the sale of IP\&S. It captures part, but not all, of the total societal value. Specifically, direct value: undervalues all IP\&S that are price regulated and/or supplied free at point of use; and; excludes 
completely the societal value of the SIS and IEA uniquely enabled by the provision of National Infrastructure.

Indirect value of infrastructure refers to the full extent of value-generating activities (uniquely enabled by, and that could not otherwise take place if national infrastructure were absent or disrupted.) Indirect value, therefore includes the value of the SIS provision and IEA enabled by infrastructure.

If indirect value is excluded when assessing the societal value of National Infrastructure is assessed, the societal value of National Infrastructure will be systemically underestimated. If National Infrastructure is undervalued, systemic underinvestment in National Infrastructure is a highly likely consequence.

Moreover, If the societal and economic value of National Infrastructure is underestimated (Systemic Perspective 10 Table 1). The societal value of ensuring National Infrastructure is a highly resilient, net zero enabling, sustainability supporting system will also be underestimated (Systemic Perspective 14 Table 1). Systemic underinvestment in the provision of these societal beneficial qualities is the highly likely consequence.

In the UK, Infrastructure owners/operators are allowed to capture a proportion of the direct value generated by the part of the National Infrastructure they operate, and are expected to reinvest the remainder in the Infrastructure systems they own/ operate. Investment in the societally beneficial systemic qualities: Net zero enabling, sustainable and Resilience adds to the societal value of National Infrastructure by creating indirect value.

However, from the owner/operator perspective, investment in these societally beneficial qualities is: 1) a direct cost; 2) generates no direct value (value they can capture or reinvest).

The value proposition of transforming National Infrastructure into a net zero enabling, sustainability supporting, resilience enhancing system is a societal one. Likewise, the opportunity cost of National Infrastructure not having these qualities. Therefore, the catalytic potential of National Infrastructure as a societal leverage point for sustainability challenges will be underexploited under business as usual approaches to Infrastructure governance, regulation and ownership. Because the societal value it generates cannot and will not be captured by individual sectors acting in isolation.

Therefore, to unlock the full catalytic potential of net zero enabling, sustainable and resilience enhancing National Infrastructure more effective mechanisms to support investment in these societally beneficial qualities is required. More specifically mechanisms for investment linked to both 1 and 2 (below) are needed:

1. The value of these qualities to National Infrastructure as a whole system not just a single owner/operator within the system and;

2. The marginal societal value enabled by National Infrastructure with these qualities compared to National Infrastructure without these qualities

In the absence of these mechanisms, the likelihood of infrastructure owners collaboratively identifying, and sharing investment in, systemically targeted interventions to enhance these desirable systemic qualities will remain low. Moreover, the level of investment in the systemic transformation of
National Infrastructure into a system that possesses the qualities will also remain low compared to the total value of the societal benefits that such investment could enable.

\section{Wicked Problem - Systemic Response}

GHG Emissions, other sustainability challenges and Resilience challenges are undesirable outcomes emerging from the normal operation of NI (Systemic Perspective 7 - Table 1). They are interdependent not just with one another (Systemic Perspective 8 Table 1), but also with the societally beneficial outcomes intentionally enabled by NI. They are deeply interdependent challenges best resolved synergistically through transformation of the wider system(s) (Figure 1) from which they emerge (Systemic Perspective 9 - Table 1).

Therefore, preventing further global warming (achieving Global Net Zero); tackling Sustainability Challenges; enhancing societal resilience to the impacts of Global warming and other Resilience Challenges are interdependent wicked system problems (Rittel and Webber, 1973; Dolan and Cosgrave, 2016) (Systemic Perspective 9 - Table 1).

\section{Climate change is a pressing and highly complex policy issue involving multiple causal factors and high levels of disagreement about the nature of the problem and the best way to tackle it. The motivation and behaviour of individuals is a key part of the solution as is the involvement of all levels of government and a wide range of non-government organisations (NGOs). (Australian Government, 2007).}

As wicked problems they must be framed and addressed in a way that is consistent with the characteristics outlined in Table 2. This will require the widespread adoption of System Thinking principles and an historic perspective to support the diagnosis of:

- Societal Leverage Points - those parts of the economy capable of catalysing society wide progress toward Net zero and other sustainability challenges (Meadows, 2009)

- System Archetypes/traps-those system structures which prevent or impede changes to system characteristics and determine the likely impact of different actions (Meadows, 2009)

- The systemic root causes and drivers of all sustainability challenges

- Synergistic, commensalistic and dissergistic opportunities

And support the identification and design of synergistic, systemically targeted interventions that exploit the above as opportunities to catalyse the necessary speed, scale and breadth of systemic transformation required to achieve Net Zero, address other Sustainability Challenges and enhance societal Resilience (Systemic Perspective 15 - Table 1).

\section{A Societally Significant Leverage Point}
National Infrastructure catalyses societal and economic multiplier effects by enabling a range of social and economic activity that simply could not occur in its absence and has the potential to facilitate the long term, sustainable, equitable, affordable realisation of 
TABLE 2 | Characteristics of and tackling wicked problems (Australian Government, 2007)

Characteristics of Wicked Problems (Australian Government, 2007:p3-5)

- Are difficult to clearly define

- Have many interdependencies and are often multi-causal

- Attempts to address wicked problems often lead to unforeseen consequences

- Are often not stable

- Usually have no clear solution

- Are socially complex

- Hardly ever sit conveniently within the responsibility of any one organisation

- Involve changing behaviour

- Some wicked problems are characterised by chronic policy failure

Tackling wicked problems (Australian Government, 2007:p35-36)

An evolving art, but one which seems to at least require:

- Holistic, not partial or linear thinking

- Innovative and flexible approaches

- The ability to work across agency boundaries

- Increasing understanding and stimulating a debate on the application of the accountability framework

- Effectively engaging stakeholders and citizens in understanding the problem and in identifying possible solutions

- Additional core skills

- A better understanding of behavioural change by policy makers

- A comprehensive focus and/or strategy

- Tolerating uncertainty and accepting the need for a long-term focus

the societally beneficial outcomes expected by the citizens and society it serves (Dolan, 2021).

National Infrastructure is a societal enabling system. It enables wider societal and economic activity (IEA). It is central to all organisational and city value chains (IEA). It supports realisation of societally beneficial infrastructure enabled outcomes (IEO). In short our Lifestyles, Societies, Cities and Economies are all emergent properties made possible by National Infrastructure (Systemic Perspective 2 - Table 1).

However, the level of GHG emissions (and hence global warming), air, water, noise pollution, solid waste and sewage produced are all also emergent properties arising from the same systems. (Systemic Perspective 7 - Table 1).

Moreover, the societal and economic impact of resilience challenges is also an emergent property arising from the resilience of National Infrastructure. (Systemic Perspective 13 - Table 1).

National Infrastructure is a societally significant leverage point capable of catalysing GHG emission reductions across all levels of society and the economy. (Systemic Perspective 16ii - Table 1).

Therefore, National Infrastructure is a societal leverage point for net zero (Systemic Perspective 16ii - Table 1). This is because the level of GHG emissions produced by the society and economy it enables are an emergent property in part determined by the characteristics of National Infrastructure (Systemic Perspective 7 - Table 1). It follows, National Infrastructure is capable of either catalysing or impeding GHG emission reductions across all levels of the society and economy it enables.

Additionally, National Infrastructure is a societal leverage point for enhanced societal resilience to the disruptive impacts of resilience challenges (Systemic Perspective 16i - Table 1). This is because highly resilient National Infrastructure can help to reduce the level of societal and economic disruption experienced by the societies and economies it enables as a consequence of
Resilience Challenges. Whereas, low resilience National Infrastructure will exacerbate the frequency, scale, intensity and duration of societal disruption caused by resilience challenges. (Systemic Perspective 13 - Table 1).

More broadly, National Infrastructure is a societal leverage point to support mitigation of the causes of, and enhance resilience to all Sustainability Challenges (Systemic Perspective 16i-iii - Table 1).

Furthermore, National Infrastructure can simultaneously be a leverage point for multiple sustainability challenges (Systemic Perspective 16iii - Table 1). Therefore, the transformation of National Infrastructure into a net zero enabling, sustainability and resilience enhancing system is an opportunity to catalyse progress toward a more Sustainable and Resilient Net Zero Economy (Dolan, 2019). (Systemic Perspective 16 - Table 1).

Moreover, the systemic transformation of National Infrastructure into a system with the qualities specified in Systemic Perspective 17 is a unilateral action that can be applied by all nations globally (Systemic Perspective 18 - Table 1).

Therefore, the transformation of National Infrastructure into a system with the qualities specified in Systemic Perspective 17 is a globally significant leverage point to support mitigation of the causes of, and enhance societal resilience to, all sustainability challenges (Systemic Perspective 18 - Table 1). The importance of which is multiplied by the projected scale of global investment in National Infrastructure - projected $\$ 3.7$ trillion/or 3.5 percent of global GDP per annum until 2040, Global Infrastructure Hub, 2017).

However, unless enabling Net Zero, enhancing resilience and addressing other sustainability challenges are made explicit priorities of National Infrastructure strategies across the globe. National Infrastructure systems are more likely to exacerbate and impede, than catalyse, progress toward Global net zero and other Sustainability Challenges (Systemic Perspective 16 - Table 1).

Therefore, whilst net zero enabling, resilience enhancing, sustainability supporting National Infrastructure cannot guarantee a net zero economy, Infrastructural inaction can all but guarantee global net zero will not be achieved. Meaning global warming will be closer to $4^{\circ} \mathrm{C}$ than $1.5^{\circ} \mathrm{C}$ meaning, National Infrastructure will need to be systemically resilient to a far greater scale, intensity and frequency of global warming driven resilience challenges.

\section{Systemic Transformation of National Infrastructure}

A sustainable, resilient, Net Zero Future requires the systemic transformation of National Infrastructure into a system that intentionally enables societally beneficial outcomes that possess the qualities Net Zero, Resilient and Sustainable. i.e., into a net zero enabling, sustainability and resilience enhancing system. (Systemic Perspectives 16 and 17 - Table 1).

This scale of systemic transformation cannot be achieved incrementally. Drawing on Meadows (2009) it will require a broad systemic focus on:

- The Mindsets from which system goals, structure and rules have emerged (Paradigms)

- The Societal Outcomes those systems are expected to enable (system goals/purpose) 
- The Governance +++ structures associated with these systems (system rules)

- The Legal Frameworks associated with these systems (system rules)

- The Mechanisms through which these systems fulfil their purpose(s) (System Structures)

(Systemic Perspective 19 - Table 1).

In particular, it will require the explicit integration of the qualities "net zero enabling," "sustainability supporting," "resilience enhancing" into:

- The stated purpose of National Infrastructure i.e., the outcomes it is expected to enable

- All Governance +++ structures that influence National Infrastructure.

(Systemic Perspective 19 - Table 1).

Where Governance +++ Structures refers to all structures that influence how we govern, regulate, manage, plan, design, procure, construct, fund, finance, own, operate, maintain, account for, value, incentivise investment in, measure the performance of, and assess future need for National Infrastructure (Dolan, 2021).

Moreover, the speed, scale and breadth of transformation needed, and the unprecedented level of interdisciplinary, cross-sectoral, society-wide collaboration requires the "Launch of a Moonshot (CST, 2020) Mission (Mazzucato, 2017; 2018a; 2018b; 2018c, 2021) tasked with establishing a diverse long-term, collaborative, dynamic, multifaceted, multi-scale, cradle-to-cradle and synergistic portfolio of systemically targeted interventions."

- A portfolio - because there is no single magic bullet solution

- Long term - because the root causes of global warming have emerged over a long term period, are deeply embedded in the systems that enable our lifestyles, societies and economies

- Transformational - because incremental approaches cannot deliver the scale, and speed of systemic transformation required.

- Diverse - because as many communities of interest must be inspired, engaged and motivated to act as possible

- Collaborative - because: the identification of leverage points, synergies, commensalisms and dissergies; and the design and implementation of systemically targeted interventions to address these will require collaboration within and between all scales of society.

- Dynamic - because net zero will require continuous action, monitoring, review and adaptation to ensure interventions are fit for purpose and sufficient in scope

- Multi-faceted - to avoid overdependence on any single intervention or intervention type

- Multi-scope - (i.e., GHG Protocol Scopes 1, 2, and 3) because action is required across a range of societal and system scales (Fong et al., 2015) at all levels of the Value chain (World Business Council for Sustainable Development, and World Resources Institute (Eds.), 2004)

- Cradle-to-Cradle - because action is needed to target emissions at all stages of the infrastructure lifecycle i.e., Capital, Operational and User emissions (British Standards Institution, 2016)

- Systemically targeted - to address the root causes of GHG emissions, by intervening at the most effective points in the system, with the most effective intervention types, at the most appropriate time

(list adapted and extended from Dolan and Street, 2019).

This Moonshot/Mission must be underpinned by a globally consistent unambiguous net zero target focused on the GHG emissions associated with both domestic production and domestic consumption. Backed by the vision, leadership and ambition of an Entrepreneurial State capable of engaging and empowering every individual and organisation who contributes to, or will be adversely affected by, Global warming. (i.e., the entire population.) (systemic perspective 20 - Table 1).

Extending the Moonshot analogy further, a global space race is needed in which all Nations launch multilateral Missions before ultimately aligning their multilateral actions in order to collaboratively achieve a Global Mission.

\section{CONCLUSION}

GHG Emissions, Sustainability Challenges, Resilience Challenges Interdependent Wicked Problems) best resolved synergistically by transforming the wider system(s) from which they emerge (systemic perspectives 7-9).

National Infrastructure is a systemically, societally, economically and globally significant synergistic leverage point for a sustainable resilient net zero future (systemic perspectives 16). The systemically targeted transformation of NI from a system that passively (unintentionally) drives the emergence of sustainability and resilience challenges into a net zero enabling, sustainability and resilience enhancing system is urgently needed to catalyse synergistic society-wide progress toward a sustainable, net zero, resilient economy (Systemic Perspective 17).

NI if transformed in this way, can:

1. Enhance (not undermine) Societal Resilience by reducing (not multiplying) the frequency, scale, intensity, duration of wider societal and economic disruption caused by resilience challenges

2. Enable (not impede) progress toward Net Zero: by catalysing (not constraining), progress toward Global Net Zero across all levels of the society, economy and value chains it enables (Figure 1).

3. Enhance (not undermine) Sustainability: by catalysing (or impeding) society-wide reductions in the levels air, water, noise pollution, solid waste and sewage produced across all levels of the society and economy it enables (Figure 1).

The Systemic transformation of NI is a unilateral action, systemic perspective 18 (Table 1). available to all nations and replicable on a global scale will require action focused on the ingredients identified in systemic perspective 19 (Table 1). Realisation of the speed, scale and breadth of transformation required will need a Mission portfolio as outlined in Systemic Perspective 20 (Table 1.) 


\section{DATA AVAILABILITY STATEMENT}

The original contributions presented in the study are included in the article, further inquiries can be directed to the corresponding author.

\section{AUTHOR CONTRIBUTIONS}

The author confirms being the sole contributor of this work and has approved it for publication.

\section{REFERENCES}

Australian Government (2007). Tackling wicked problems: A public policy perspective. Australian Public Service Commission. Available at: https:// legacy.apsc.gov.au/tackling-wicked-problems-public-policy-perspective.

Beckford, J. (2009). An Overview of Systemic Interdependencies of the UK National Infrastructure.

British Standards Institution (2016). Carbon Management in Infrastructure. London, United Kingdom: BSI.

Carhart, N., and Rosenberg, G. (2016). A Framework for Characterising Infrastructure Interdependencies. International Journal of Complexity in Applied Science and Technology 1 (1), 35-60. doi:10.1504/ijcast.2016.081294

CST (2020). Principles for Science and Technology Moon-Shots: Letter from Council for Science \& Technology to Prime Minister (P. 5). The Council for Science and Technology. Available at: https://assets.publishing.service.gov.uk/ government/uploads/system/uploads/attachment_data/file/896786/20200625 CSTLetteronMoon-shotsPrinciples.pdf.

Dolan, T., and Cosgrave, E. (2016). Aligning Systemic Infrastructure Decisions with Social Outcomes. Proc. Inst. Civil Eng. - Civil Eng. 169 (4), 147. doi:10.1680/jcien.2016.169.4.147

Dolan, T. (2017). National Infrastructure Commission Digitally Connected Infrastructure System Resilience: Literature Review (UCL) (P. 61) [Literature Review]. NIC. Available at: https://www.nic.org.uk/publications/literaturereview-resilience-digitally-connected-infrastructure-systems/.

Dolan, T. (2018). Resilience Shift: Guest Blog. The Role of Economic Infrastructure in Limiting Global Warming. Available at: https://www.resilienceshift.org/roleof-economic-infrastructure-in-limiting-global-warming/.

Dolan, T. (2021). "Resilient Infrastructure Systems," in The Intelligent Nation: How to Organise a Country (Oxfordshire, England, UK: Routledge), 116-123. Available at: https://www.ukcric.com/media/1404/systemically-resilientnational-infrastructure-extract-from-the-intelligent-nation-mar21.pdf.

Dolan, T., and Street, R. (2019). Interdependence Analysis for Systemically Resilient Infrastructure systemsPlacing Resilience at the Heart of Systemic Infrastructure Decision-Making Processes (CIRIA Briefing Ref: 09-01-191 B; P. 14). Available at: https://www.ciria.org/Briefings/interdependence_resilience_briefing.aspx.

Dolan, T. (2019). Successful Cities Rely on Resilient Infrastructure [Global NonProfit/Thirdsector]. Successful Cities Rely on Resilient Infrastructure. Available at: https://newcities.org/the-big-picture-successful-cities-relyresilient-infrastructure/.

Dolan, T. (2020). Systemically Resilient Prosperity: Adapting to and Learning from COVID-19 (And Other Strategic Challenges) [Research]. Available at: https:// www.ukcric.com/insights/systemically-resilient-prosperity-adapting-to-andlearning-from-covid-19-and-other-strategic-challenges/.

Edwards, P. N. (2003). "Force, Time, and Social Organization in the History of Sociotechnical Systems," in Modernity and Technology: The Vol. Draws on an Internat. Workshop Held at the Univ. Of Twente in the Netherlands in November 1999. Editors T. J. Misa, P. Brey, and A. Feenberg (Cambridge: MIT Press).

Figueres, C., and Rivett-Carnac, T. (2020). Future We Choose: Surviving the Climate Crisis. Available at: https://www.overdrive.com/search?q=17CC75C148DB-411E-967E-DD0C86CEE0EB.

Fong, W. K., Sotos, M., Doust, M., Schultz, S., Marques, A., and Deng-Beck, C.World Resources Institute (2015). Global Protocol for Community-Scale Greenhouse Gas Emission Inventories: An Accounting and Reporting

\section{FUNDING}

The research published here builds on research undertaken as part of the International Centre for Infrastructure Futures (ICIF EPSRC and ESRC grant reference EP/K012347/1) and was undertaken as part of UKCRIC Collaboration Node (EPSRC Grants reference EP/R017727/1). Both of which were made possible by the inspirational vision and leadership of Professor Brian Collins.

Standard for Cities. Washington, D.C., United States: World Resources Institute. Available at: https://www.deslibris.ca/ID/246277.

Global Infrastructure Hub (2017). Global Infrastructure Outlook (Global Infrastructure Outlook, P. 212). Global Infrastructure Hub. Available at: https://www.oxfordeconomics.com/publication/open/283970.

Helm, D. (2020). Net Zero: How We Stop Causing Climate Change. Chichester, United Kingdom: William Collins.

Hollnagel, E. (2014). Resilience Engineering and the Built Environment. Building Res. Inf. 42 (2), 221-228. doi:10.1080/09613218.2014.862607

Hughes, T. P. (1989). The Evolution of Large Technological Systems. The Social Construction of Technological Systems: New Directions in the Sociology and History of Technology. Cambridge, MA, United States: MIT Press.

IPCC (2018). IPCC 2018: Global Warming of $1.5^{\circ} \mathrm{C}$. An IPCC Special Report on the Impacts of Global Warming of $1.5^{\circ} \mathrm{C}$ above Pre-industrial Levels and Related Global Greenhouse Gas Emission Pathways, in the Context of Strengthening the Global Response to the Threat of Climate Change, Sustainable Development, and Efforts to Eradicate Poverty. IPCC. Available at: https:// www.ipcc.ch/sr15/download/\#chapter.

Mazzucato, M. (2017). Mission-oriented Innovation Policy: Challenges and Opportunities (Working Paper IIPP WP 2017-01; IIPP Working Papers, P. 42). UCL. Available at: https://www.ucl.ac.uk/bartlett/public-purpose/sites/ public-purpose/files/moip-challenges-and-opportunities-working-paper-20171.pdf.

Mazzucato, M. (2018a). Mission-oriented Research \& Innovation in the European Union (1st ed.). European Commission. Available at: https://publications. europa.eu/en/publication-detail/-/publication/5b2811d1-16be-11e8-925301aa75ed71a1/language-en.

Mazzucato, M. (2021). Mission: Economics: A Moonshot Approach to the Economy. Northwood, United Kingdom: Allen Lane.

Mazzucato, M. (2018b). The Entrepreneurial State: Debunking Public vs. Private Sector Myths. London: Penguin Books.

Mazzucato, M. (2018c). The Value of Everything: Making and Taking in the Global Economy. Northwood, United Kingdom: Allen Lane, an imprint of Penguin Books.

Meadows, D. H. (2009). in Thinking in Systems: A Primer. Editor D. Wright. Available at: https://wtf.tw/ref/meadows.pdf.

Oughton, E. J., Usher, W., Tyler, P., and Hall, J. W. (20182018). Infrastructure as a Complex Adaptive System. Complexity 2018, 1-11. doi:10.1155/2018/3427826

Perrow, C. (2011). Normal Accidents: Living with High Risk Technologies. Available at: https://www.scopus.com/inward/record.uri?eid=2-s2.084890664445\&partnerID=40\&md5=e616aa6bd97f92d2ec3ca9d73ccdbb88.

Punzo, G., Tewari, A., Butans, E., Vasile, M., Purvis, A., Mayfield, M., et al. (2020). Engineering Resilient Complex Systems: The Necessary Shift toward Complexity Science. IEEE Syst. J. 14 (3), 3865-3874. doi:10.1109/ JSYST.2019.2958829

Rinaldi, S. M., Peerenboom, J. P., and Kelly, T. K. (2001). Identifying, Understanding, and Analyzing Critical Infrastructure Interdependencies. IEEE Control. Syst. 21 (6), 11-25. doi:10.1109/37.969131

Rittel, H. W. J., and Webber, M. M. (1973). Dilemmas in a General Theory of Planning. Policy Sci 4 (2), 155-169. doi:10.1007/bf01405730

Rosenberg, G., Carhart, N., Edkins, A. J., and Ward, J. (2014). Development of a Proposed Interdependency Planning and Management Framework [Report]. Int. Centre Infrastructure Futures. Available at: http://discovery.ucl.ac.uk/ 1455020/. doi:10.14324/20141455020 
Royal Academy of Engineering (Great Britain) \& Engineering the Future (Organization) (2011). Infrastructure, Engineering and Climate Change Adaptation: Ensuring Services in an Uncertain Future. Royal Academy of Engineering, on Behalf of Engineering the Future. Available at: https://www. raeng.org.uk/publications/reports/engineering-the-future.

Treasury, H. M. (2017). National Infrastructure Commission Framework Document.. London, United Kingdom: HM Treasury.

Varga, L., Grubic, T., Greening, P., Varga, S., Camci, F., and Dolan, T. (2014). Characterizing Conversion Points and Complex Infrastructure Systems: Creating a System Representation for Agent-Based Modeling. Complexity 19 (6), 30-43. Scopus. doi:10.1002/cplx.21521

World Business Council for Sustainable Development, \& World Resources Institute (Eds.) (2004). The Greenhouse Gas Protocol: A Corporate Accounting and Reporting Standard (Rev. Ed). Washington, D.C., United States: World Business Council for Sustainable Development; World Resources Institute.
Conflict of Interest: The author declares that the research was conducted in the absence of any commercial or financial relationships that could be construed as a potential conflict of interest.

Publisher's Note: All claims expressed in this article are solely those of the authors and do not necessarily represent those of their affiliated organizations, or those of the publisher, the editors and the reviewers. Any product that may be evaluated in this article, or claim that may be made by its manufacturer, is not guaranteed or endorsed by the publisher.

Copyright (C) 2021 Dolan. This is an open-access article distributed under the terms of the Creative Commons Attribution License (CC BY). The use, distribution or reproduction in other forums is permitted, provided the original author(s) and the copyright owner(s) are credited and that the original publication in this journal is cited, in accordance with accepted academic practice. No use, distribution or reproduction is permitted which does not comply with these terms. 